\title{
Metastatic Colorectal Adenocarcinoma of the Mastoid Bone: A Rare Case Report
}

\author{
Neil Mundi ${ }^{1}$, Stefan Hamilton ${ }^{1}$, Bret Wehrli², Lorne S Parnes ${ }^{1}$ \\ ${ }^{1}$ Department of Otolaryngology - Head and Neck Surgery and ${ }^{2}$ Department of Pathology, London Health Sciences Center, \\ London, $\mathrm{ON}$.
}

\section{Corresponding Author: \\ Dr. Neil Mundi \\ Email: neilmundi@gmail.com}

This is an Open Access article distributed under the terms of the Creative Commons Attribution License (creativecommons.org/ licenses/by/3.0).

Received : March 18, 2019

Accepted : June 29, 2019

Published : July 10, 2019

\begin{abstract}
Background: Metastases to the temporal bone are exceedingly rare, representing $<0.2 \%$ of all head and neck tumors. The most common primary malignancies are from breast, lung, prostate and skin. Given the rarity of temporal bone metastases and paucity of such case reports in the literature, the dissemination of a diagnostic approach is a worthwhile venture. Case Report: Here we describe a patient with known metastatic adenocarcinoma of the descending colon presenting with unilateral aural fullness, hearing loss and ataxia. Radiographic investigations revealed a soft tissue mass within the mastoid bone, and biopsy confirmed metastatic colorectal adenocarcinoma. The patient's disease progressed over time, causing facial paralysis, and eventually her demise. Conclusion: This unique and uncommon case highlights the importance of thoroughly investigating patients with known malignancy who present with unilateral otologic and vestibular symptoms.
\end{abstract}

Keywords: Adenocarcinoma, Biopsy, Hearing Loss, Mastoid, Temporal Bone.

\section{Introduction}

Metastases to the temporal bone are exceedingly rare, representing $<0.2 \%$ of all head and neck tumors. Gloria-Cruz et al. examined the prevalence of metastases to the temporal bone in 212 patients with primary non-disseminated malignant neoplasms. The colon was amongst the least common types, with only $2.1 \%$ of patients having it as their primary site of malignancy. Among histopathologic subtypes, adenocarcinoma was the most common type of malignancy found to metastasize to the temporal bone [1]. When delineating the metastatic areas within the temporal bone, the most common were the petrous apex, mastoid, internal auditory canal and middle ear. Presenting symptoms of temporal bone metastases vary but include hearing loss, vertigo or dizziness, facial weakness and tinnitus. It should however be noted that many patients are asymptomatic even when their disease burden is high [1].
Reports of metastatic colorectal adenocarcinoma to the temporal bone, as expected given the aforementioned information, are few, with only a handful of cases reported in the last 60 years [2-6]. Within this case report we describe a patient with known metastatic adenocarcinoma of the descending colon who presented with unilateral aural fullness, hearing loss and ataxia.

\section{Case Report}

A 64 year old female initially presented to her physician with abdominal pain and elevated liver enzymes. Ultrasound and subsequent biopsy of the liver revealed metastatic adenocarcinoma. Colonoscopy was performed and revealed the primary tumor within the descending colon. Further diagnostic workup at the time of presentation did not demonstrate any other metastatic deposits. The patient's disease was deemed unresectable and she was initiated on palliative chemotherapy of 
FOLFIRI and bevacizumab, which she tolerated well.

Approximately one year after initiating chemotherapy, the patient presented to her local emergency department with left sided aural fullness, hearing loss, ataxia and positional vertigo. The patient had no previous history of any otologic or vestibular conditions. A CT scan of the head [Fig.1] demonstrated an ill-defined, destructive mass within the left petrous bone. Subsequent MRI of the head revealed a $2.3 \mathrm{~cm} \times$ $2.3 \mathrm{~cm} \times 2.1 \mathrm{~cm}$ soft tissue mass in the left mastoid anterior to the sigmoid sinus and abutting the internal jugular vein [Fig.2]. Our initial assessment demonstrated right sided posterior semicircular canal benign paroxysmal positioning vertigo (BPPV) as the cause for the patient's vertigo. Physical examination otherwise revealed a normal otoscopic examination and no facial weakness. The audiogram revealed normal low to mid frequency hearing on the right with a sloping moderate-severe sensorineural hearing loss. On the left, there was normal low to mid frequency hearing sloping to a severe high frequency sensorineural hearing loss. In addition, there was an elevated speech reception threshold on the left of $20 \mathrm{~dB}$ with $88 \%$ word recognition compared to $100 \%$ on the right. Given the concerning radiographic findings and the rarity of metastatic colon cancer to the temporal bone, the patient was offered biopsy and underwent a mastoidectomy with biopsy. Intra-operatively a fleshy mass was noted and pathologic analysis revealed metastatic colorectal adenocarcinoma, confirmed with CDX2 staining [Fig.3,4]. CK 20 staining was also performed and was consistent with metastatic colorectal adenocarcinoma.

On post-operative day two the patient developed a left sided facial paralysis, HouseBrackmann grade four. Systemic corticosteroids were ineffective and the patient's paralysis persisted. Over time the patient developed progressive dysphagia and weight loss. She was deemed unfit for further chemotherapy. Repeat

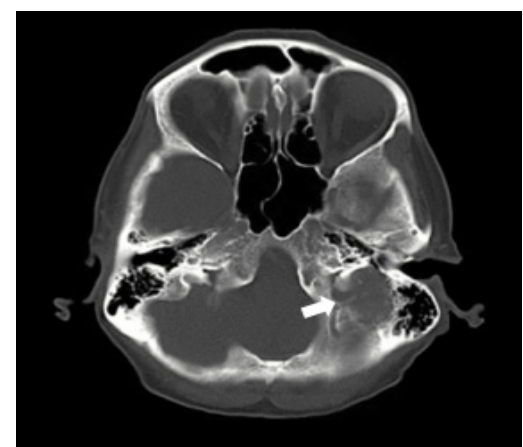

Fig.1: CT head demonstrating ill-defined left temporal bone lesion (arrow).

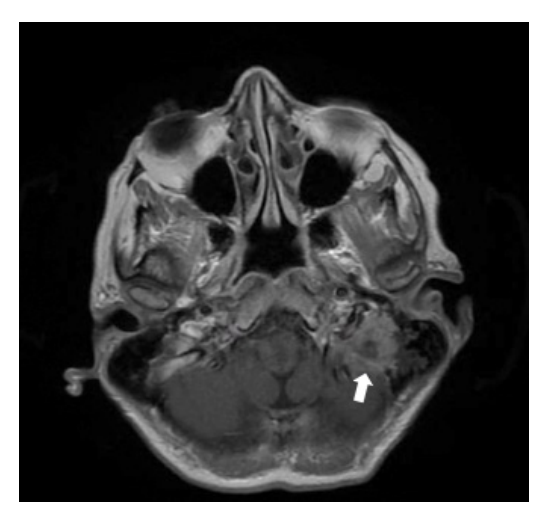

Fig.2: T1-weighted MRI of the skull base demonstrating a homogenous, enhancing mass involving the left mastoid bone (arrow). Scan was performed without Gadolinium.

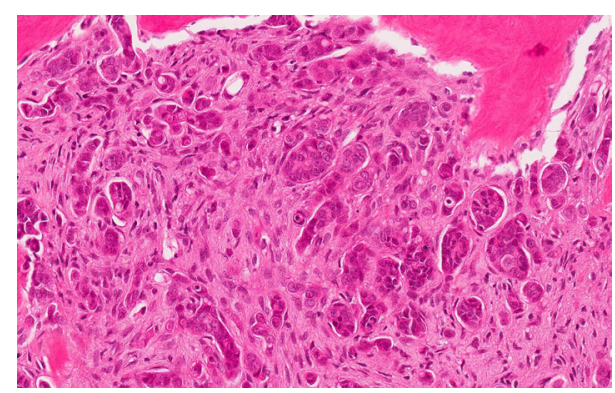

Fig.3: H\&Estaining ofmastoidmass reveals adenocarcinoma.

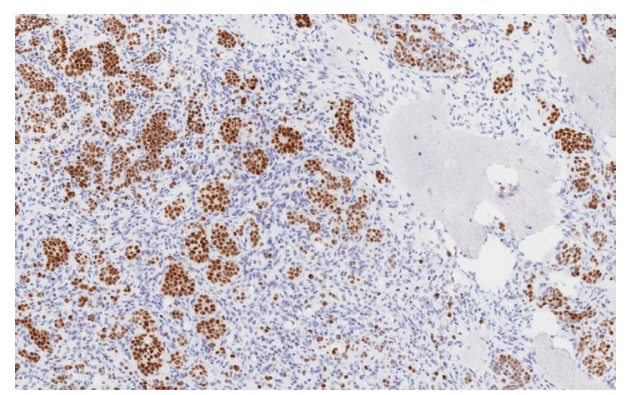

Fig.4: CDX2 staining of mastoid mass confirms metastatic adenocarcinoma. 
MRI of the head two months after surgery demonstrated enlargement of the mass at $4.3 \mathrm{~cm}$ $\times 3.8 \mathrm{~cm}$ with compression of the sigmoid sinus and internal jugular vein [Fig.5]. In addition, there was significant narrowing of the jugular foramen and hypoglossal canal. Palliative radiotherapy to the temporal bone at 30 Gy over 10 fractions was performed.

The patient's symptoms continued to progress and she was admitted to her local hospital for end of life care. She succumbed to her disease four months after the diagnosis of temporal bone metastasis.

\section{Discussion}

Metastases to the mastoid bone from adenocarcinoma of the colon are exceedingly rare, and few cases have ever been reported [26]. As such, relatively little is known regarding its presentation, natural history and optimal treatment methods. Various types of malignancies metastasize to the temporal bone including solid malignancies (lung and breast most commonly) [1,7] and hematologic malignancies such as leukemia [8]. The route of metastatic spread to the temporal bone is primarily hematologic; however, direct extension, meningeal carcinomatosis and leukemic infiltration can also occur [9]. A recent investigation by Song et al. described the clinical characteristics of temporal bone metastases. The authors found that the median duration from diagnosis of primary malignancy to temporal bone metastases was 22.5 months. In stark contrast, the median time to death from the diagnosis of temporal bone metastases was 3 months [10], highlighting the advanced stage of disease associated with this entity. In our patient, death occurred four months after the diagnosis of temporal bone metastasis. The largest series to date examining metastases to the temporal bone demonstrated that a significant proportion of patients are asymptomatic [1]. However, hearing loss, pain and facial paralysis have been described as presenting symptoms [10]. There can also be

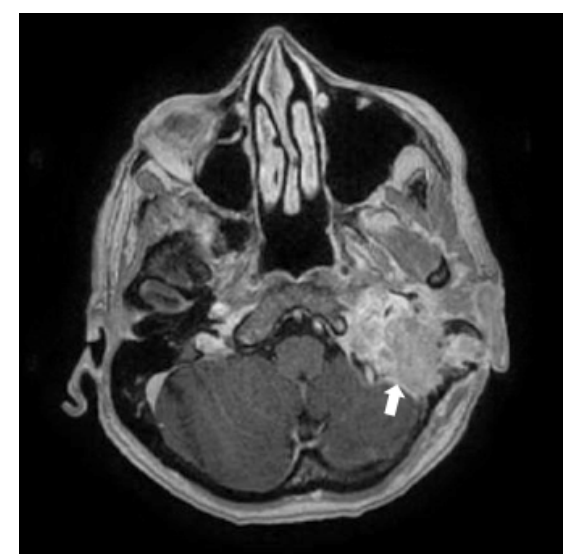

Fig.5: Contrast enhanced MRI head revealing an enlarged mass within the left mastoid bone with compression of surrounding structures (arrow).

manifestations of other pathologies such as chronic otitis media, and temporal bone metastases can sometimes masquerade as more benign entities. Our patient coincidentally had BPPV, but of the contralateral ear, and it was successfully treated with the particle repositioning maneuver.

Diagnostic assessment for temporal bone metastases should include a detailed history and physical examination, audiogram, and CT/MRI of the temporal bone. Treatment of our patient's metastasis was palliative using radiotherapy. This is reflective of the treatments that most individuals receive given the advanced stage of their disease at the time of diagnosis of temporal bone metastases [10].

\section{Conclusion}

Metastasis to the temporal bone is a rare clinical entity and represents a very small percentage of head and neck tumors. Patients with temporal bone metastasis have a poor prognosis as they tend to have very advanced disease. Here we describe a patient with biopsy-proven metastatic adenocarcinoma of the descending colon to the mastoid bone, one of only a few such case reports in the literature to date. Treatment options for these patients are limited to attempts at systemic treatment and local radiation as surgery is high-risk 
and unlikely to yield significant clinical benefit. Presenting symptomology can overlap with other, more common pathologies and as such, it is important to consider temporal bone metastases in symptomatic individuals with a history of an advanced primary malignancy.

Contributors: NM \& SH: Literature review, development of manuscript; BW: provided histopathological images, edited manuscript. LP provided radiographic images, oversaw development of manuscript, edited manuscript. NM will act as a study guarantor. All authors approved the final version of this manuscript.

Funding: None; Competing interests: None stated.

\section{References}

1. Gloria-Cruz TI, Schachern PA, Paparella MM, Adams GL, Fulton SE. Metastases to temporal bones from primary non systemic malignant neoplasms. Arch Otolaryngol Head Neck Surg. 2000;126):209-214

2. Merrick Y. Metastatic colon adenocarcinoma of the middle ear. Arch Otol. 1983;238:103-105.
3. Frisch CD, Breen JT, Duckworth EA, Sweeney AD. Colon cancer metastasis to the lateral skull base masquerading as mastoiditis. J Neurol Surg B. 2017;78 (S01):S1-S156.

4. Levine PA, Ruah CB, Bohigian K, Vincent ME, Vaughan CW. Metastatic sigmoid colon adenocarcinoma to the temporal bone. Otol HN Surg. 1987;97:500-503.

5. Hill BA, Kohut RI. Metastatic adenocarcinoma of the temporal bone. Arch Otol. 1976;102:568-571.

6. Jorgensen M. Metastatic carcinoma of the temporal bone. J Laryn Otol. 1961;75:513-518.

7. Streitman MJ, Sismanis A. Metastatic carcinoma of the temporal bone. Am J Otol. 1996;17:780-783.

8. Aljafar HM, Alsuhibani SS, Alahmari MS, Alzahrani MA. Temporal bone metastasis as a sign of relapsing chronic lymphocytic leukemia. Saudi Med J. 2015;36:12331235.

9. Berlinger NT, Koutroupas S, Adams G, Maisel R. Patterns of involvement of the temporal bone in metastatic and systemic malignancy. Laryngoscope. 1980;90:619-627.

10. Song K, Park KW, Heo JH, Song IC, Park YH, Choi JW. Clinical characteristics of temporal bone metastases. Clin Exp Otol. 2019;12:27-32. 